J. Phys. IV France 138 (2006) 245-250

(C) EDP Sciences, Les Ulis

DOI: $10.1051 / \mathrm{jp} 4: 2006138028$

\title{
Microscopie interférentielle X-UV : un outil pour l'étude des endommagements des surfaces optiques
}

\author{
G. Jamelot ${ }^{1}$, D. Ros ${ }^{1}$, K. Cassou ${ }^{1}$, S. Kazamias ${ }^{1}$, A. Klisnick ${ }^{1}$, M. Kozlová², \\ T. Mocek ${ }^{2}$, P. Homer ${ }^{2}$, J. Polan², M. Stupka² et B. Rus ${ }^{2}$ \\ ${ }^{1}$ LIXAM, Laboratoire d'Interaction du Rayonnement $X$ avec la Matière, UMR 8624, \\ Bât. 350, Campus de l'Université Paris XI, 91405 Orsay Cedex, France \\ ${ }^{2}$ Institute of Physics, Pals Centre, Prague 8, Czech Republic
}

\begin{abstract}
Résumé. Nous présentons des résultats récents concernant des premières investigations de microscopie interférentielle par laser X-UV d'endommagement optique. Le laser X-UV utilisé est un laser collisionnel en régime quasi-stationnaire émettant à $21.2 \mathrm{~nm}$, développé au Prague Asterix Laser System (PALS, Prague, République Tchèque). Des échantillons de silice fondue de haute qualité, avec ou sans rayure, étaient irradiées en face avant par un laser bleu, correspondant au $3^{\text {ème }}$ harmonique du laser à iode du PALS $(1.315 \mu \mathrm{m})$, servant également à réaliser le laser X-UV à $21.2 \mathrm{~nm}$. Celui-ci était utilisé, $5 \mathrm{~ns}$ après l'irradiation pour réaliser une imagerie microscopique et interférentielle de la face arrière de l'échantillon. Les résultats font apparaître des déformations locales transitoires. Des premières analyses mettent en évidence une probable variation de la rugosité de la surface. Cette démonstration expérimentale encourageante ouvre la voie à de futures investigations, notamment sur notre prochaine installation laser : LASERIX.
\end{abstract}

\section{INTRODUCTION}

Les lasers X-UV peuvent être utilisés pour des expériences d'application, soit comme source d'irradiation avec possibilité d'être focalisée, soit comme source d'imagerie en utilisant des techniques d'interférométrie. Ces dernières ont donné des résultats très prometteurs à la fin des années 90 . Ainsi par exemple, au Laboratoire de Spectroscopie Atomique et Ionique (LSAI, ancienne dénomination du LIXAM), en collaboration avec l'Institut d'Optique Théorique et Appliquée (IOTA), nous avons développé en 1996 un interféromètre à division de front d'onde à double miroir de Fresnel. Il a été testé en 1997 avec le laser X-UV émettant à $21.2 \mathrm{~nm}$ [1], permettant ainsi d'obtenir des interférogrammes d'échantillon test (marche de $50 \mathrm{~nm}$ ). Cet interféromètre a ensuite permis d'étudier la "migration" de défaut sur une anode de niobium en présence de champ électrique intense appliqué à la surface de l'anode [2, 3], en collaboration avec le CEA-DAPNIA au Laboratoire pour l'Utilisation des Lasers Intenses (LULI) en France. Des investigations plus récentes, que nous avons mené au PALS [4], en collaboration avec l'équipe de B. Rus, ont permis de compléter nos premières études [5, 6]. Ces différentes recherches nous ont ainsi permis d'accumuler une grande expérience en matière de technique interférométrique par laser X-UV. L'étape suivante a consisté à coupler l'interféromètre à un miroir imageur permettant ainsi de disposer d'une microscopie interférentielle, particulièrement adaptée pour décrire in situ des processus nécessitant une résolution spatiale à l'échelle micrométrique et temporelle à l'échelle de la durée de l'impulsion laser X-UV. Nous avons notamment démontré l'intérêt d'un tel outil dans le cadre d'expériences de sonde de plasmas denses créés par laser [7].

C'est donc sur la base de cette technique que nous avons étudié l'état de surfaces optiques soumises à des irradiations sous champ laser. L'intérêt de ces investigations est d'étudier qualitativement et quantitativement la tenue au flux des surfaces optiques fonctionnant en transmission. Celles-ci sont particulièrement sensibles dans les installations laser de très grande puissance, comme le laser MégaJoules (LMJ), en construction actuellement en Aquitaine. Ainsi si l'on constate que les seuils de destruction sont de l'ordre de $30 \mathrm{~J} / \mathrm{cm}^{-2}$ (notamment pour des lames de verre en silicate), les processus 

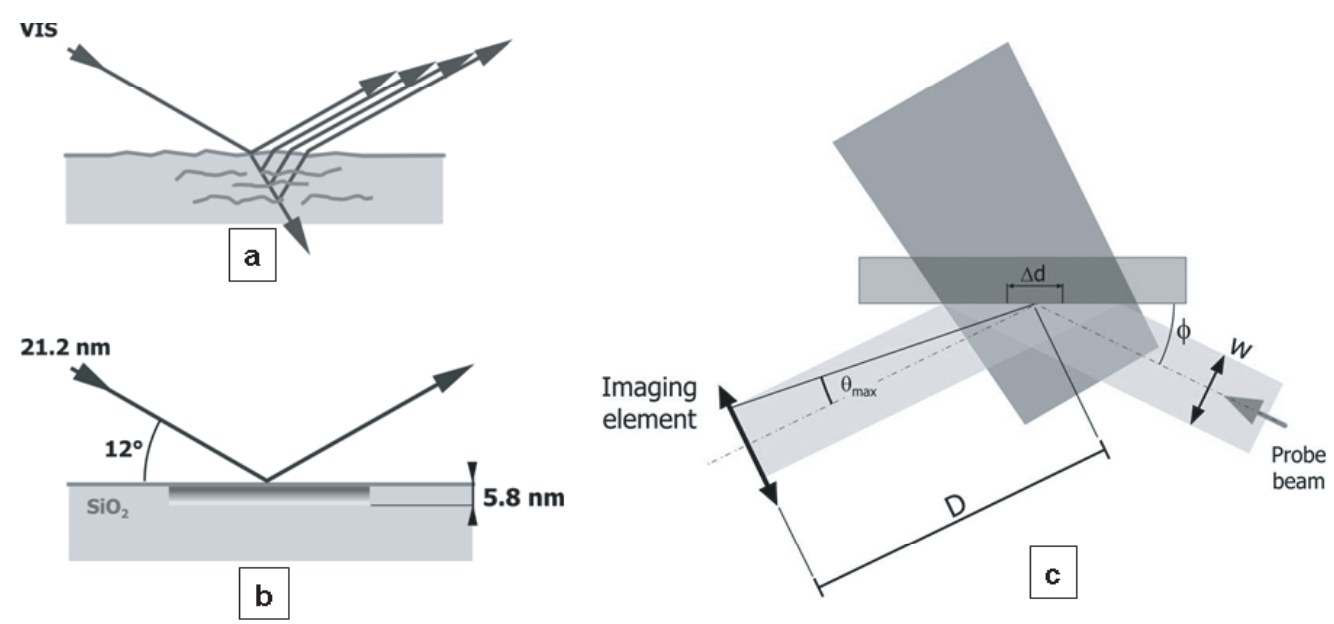

Figure 1. Schémas de principe d'utilisation d'un laser en incidence rasante, émettant dans le domaine visible (a) et dans le domaine X-UV (b) pour sonder une surface. Schéma de principe représentant à la fois l'irradiation d'une surface optique par un laser "d'endommagement" et son inspection en face arrière avec le faisceau laser X-UV (faisceau sonde).

aboutissant à la destruction ne sont pas très bien connus. En particulier, il a été souvent remarqué que les destructions apparaissaient en face arrière de lames irradiées par le laser, ou en volume.

Le fait d'utiliser un laser X-UV $(21.2 \mathrm{~nm})$ constitue un atout majeur dans la problématique qui nous intéresse (voir figure 1-a et 1-b). En effet, le laser X-UV doit être utilisé sous incidence rasante et travaille en conséquence dans le régime de réflexion totale, contrairement aux lasers du domaine visible qui sont partiellement réfractés par le milieu et peuvent être diffusés par les défauts "en volume". Au contraire, la profondeur de pénétration du laser X-UV est très faible (inférieure à $6 \mathrm{~nm}$ ), ce qui permet de sonder uniquement la face arrière de l'échantillon irradié par le faisceau laser bleu (voir figure 1-c).

Par ailleurs, l'utilisation d'un laser X-UV permet d'atteindre une résolution spatiale élevée. En,effet, la résolution " $\Delta d \sin \phi$ " est directement liée à la longueur d'onde du laser utilisé par la relation suivante :

$$
\Delta d \sin \phi=\frac{\lambda}{N . A .}=\frac{\lambda}{\sin \theta_{\max }} \cong \frac{\lambda}{w} 2 D,
$$

où les différents paramètres représentés en figure 1-c. Ainsi dans le cas d'un laser émettant à $500 \mathrm{~nm}$, on arrive à une résolution de $10 \mu \mathrm{m}$ alors que dans le cas du laser X-UV utilisé dans nos expériences, la résolution était de $0.4 \mu \mathrm{m}$.

\section{CONFIGURATION EXPÉRIMENTALE}

L'arrangement expérimental de l'enceinte d'application correspondant à l'irradiation des surfaces est montré en figure 2. L'échantillon est irradié sur une surface d'environ $1 \mathrm{~mm}^{2}$ par un faisceau laser bleu émettant à $438 \mathrm{~nm}$ pendant une durée de $300 \mathrm{ps}$. Ce laser est intrinsèquement synchronisé avec le laser infrarouge de pompe du laser XUV, car ils sont tous deux issus d'un seul et même oscillateur. L'investigation temporelle de l'évolution de la surface est obtenue simplement à l'aide d'une ligne à retard optique entre les deux faisceaux.

Le faisceau laser X-UV est réalisé dans une autre enceinte, située à environ 2.5 mètres de l'échantillon. Ce laser à ions néonoïdes de zinc fonctionne en double passage $[4,8]$, ce qui lui assure d'excellentes qualités optiques. Il n'est malheureusement obtenu qu'à très faible cadence (20 minutes 


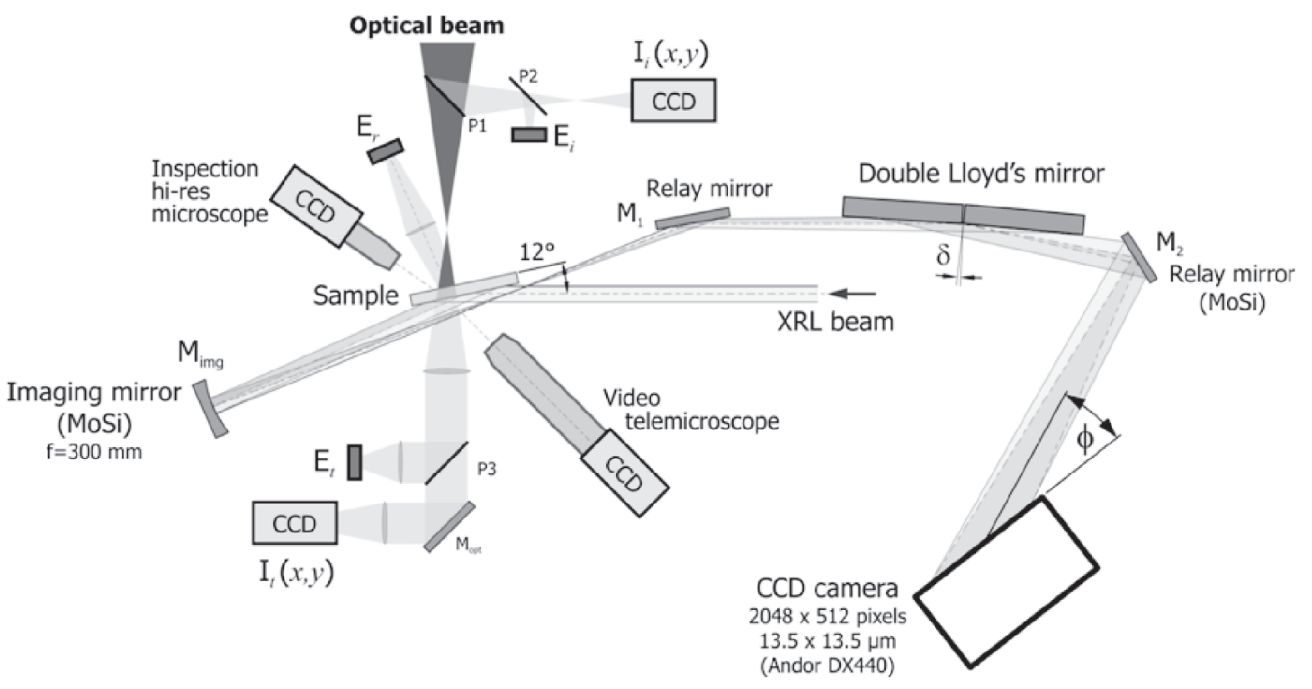

Figure 2. Schéma de dispositif expérimental pour la microscopie interférentielle X-UV. L'échantillon ("sample") est irradié par le laser d'endommagement. Le laser X-UV est réfléchi par l'échantillon, puis par le miroir imageur (grandissement 8), enfin par le bi miroir de Fresnel (noté "double Lloyd's mirror" sur la figure). Plusieurs caméras, systèmes microscopiques de visée in-situ de l'échantillon et de calorimètres permettent de mesurer les énergies transmises et réfléchies par l'échantillon.

au minimum entre deux tirs). Le faisceau XUV est ensuite envoyé sous un angle d'attaque de $12^{\circ}$ sur la face arrière de l'échantillon. Puis le système optique composé d'un miroir sphérique imageur en Mo:Si couplé à un interféromètre à bi-miroir de Fresnel (noté "Double Lloyd" sur la figure) permet d'obtenir dans le plan du détecteur CCD une image interférométrique de la zone de l'échantillon sondé par le faisceau laser X-UV.

En complément de l'interféromètre imageur, différents diagnostics (calorimètres, microscopes couplés à des caméras CCD) sont disposés autour de l'échantillon (voir figure 2). Ce type de matériels aboutit à un encombrement expérimental notable, comme on peut le constater sur la photographie de la figure 3, nécessitant en conséquence une phase de réglage importante. L'ensemble des diagnostics installés permet donc d'enregistrer simultanément l'état de surface en face arrière, ainsi que des données concernant les énergies transmises et réfléchies par l'échantillon irradié, particulièrement utiles à croiser dans l'analyse finale de l'évolution de l'échantillon.

\section{RÉSULTATS EXPÉRIMENTAUX ET PREMIÈRES ANALYSES}

La résolution de ce dispositif a été mesurée à l'aide d'un substrat de silice fondue de planéité $\lambda / 20$ à $632 \mathrm{~nm}$, utilisé comme surface de référence, sans laser d'endommagement. La résolution spatiale, limitée par la taille des pixels du détecteur, est de l'ordre de $2 \mu \mathrm{m}$ sur la surface étudiée, et l'interfrange correspond à une différence d'altitude de $50 \mathrm{~nm}$.

L'étude d'un cas expérimental donné nécessite l'enregistrement de trois interférogrammes successifs avec des faisceaux laser X-UV identiques: le premier enregistré avant le passage du laser d'endommagement, le second au passage de ce laser (durant ces expériences, le retard du laser X-UV était de $5 \mathrm{~ns}$ ); enfin, un troisième enregistrement permet d'observer l'état de la surface après l'interaction et les éventuels dommages. La fluence d'irradiation sur les surfaces a varié au cours de cette série d'expériences de 5 à $50 \mathrm{Jcm}^{-2}$, c'est-à-dire de part et d'autre du seuil de dommage.

La figure 4 montre un ensemble typique de trois interférogrammes de la face arrière d'une lame de silice. La partie supérieure de la figure est l'image interférométrique de la surface non perturbée, 


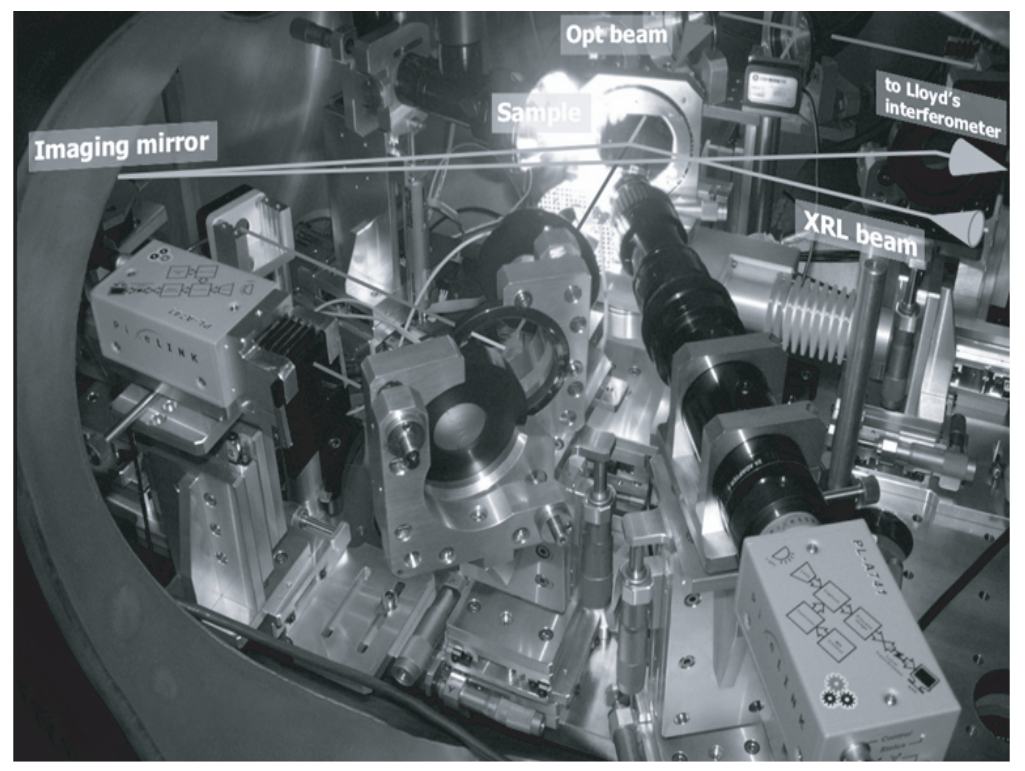

Figure 3. Image représentant l'ensemble des dispositifs expérimentaux disposés dans l'enceinte d'application.

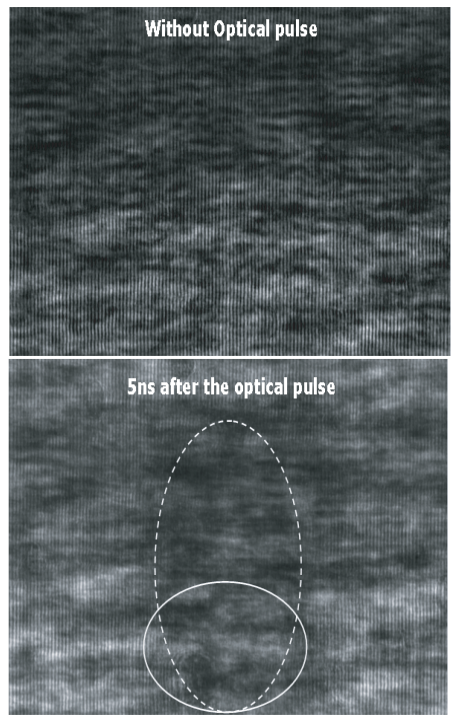

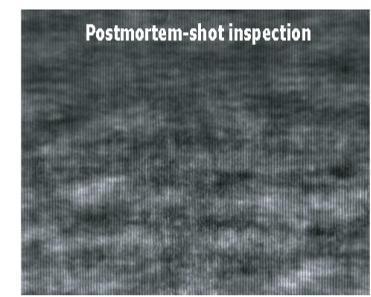

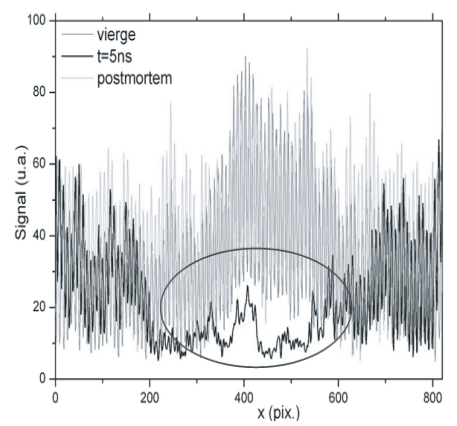

Figure 4. Interférogrammes X-UV d'une surface optique. Du haut en bas : sans irradiation, puis $5 \mathrm{~ns}$ après le passage du laser d'endommagement, enfin enregistrement "post mortem" 20 minutes plus tard. En bas, tracés des signaux pour les trois interférogrammes correspondant à la zone entourée d'un trait blanc continuu.

avant toute irradiation. L'interférogramme suivant correspond au passage du laser d'endommagement, avec une fluence d'irradiation de $10 \mathrm{Jcm}^{-2}, 5 \mathrm{~ns}$ avant le passage du laser X-UV. On constate que la réflectivité de la surface a considérablement diminué et que les franges d'interférence ont quasiment disparu sur toute la surface du faisceau d'endommagement. La surface irradiée a été analysée ensuite sans laser d'endommagement, lors du tir suivant du laser X-UV, environ 20 minutes après. On constate que les franges sont en grande partie restaurées et que la forte perturbation de la surface causée par le 
laser d'endommagement est transitoire à ce niveau de fluence. Les dommages révélés par ce dernier interférogramme sont très faibles et peu étendus. Le graphe du bas de la figure 4, qui montre la superposition de ces 3 interférogrammes, confirme la quasi-disparition de la réflectivité 5 ns après le passage du laser et la restauration presque complète de la surface ensuite.

\section{CONCLUSIONS ET PERSPECTIVES}

Ces résultats inattendus nécessitent une analyse détaillée pour être correctement interprétés. Il faut faire appel à un phénomène rapide, à l'échelle de la nanoseconde, qui peut être généré pour des fluences inférieures au seuil de dommage. Plusieurs hypothèses peuvent être avancées, effet thermique, onde de choc... Mais il faudrait disposer de beaucoup plus de résultats que nous avons pu en récolter au cours de ces expériences au PALS.

En particulier une analyse temporelle détaillée montrant l'évolution de la perturbation en fonction du retard du laser X-UV de 0 à 5 ns et au-delà est absolument nécessaire. Mais cette analyse demanderait un nombre de tirs laser tel qu'il est peu probable de pouvoir la réaliser au PALS.

Au contraire LASERIX [10, 11], plateforme de l'Université Paris-Sud 11, qui sera opérationnelle à partir de l'été 2007 sur le site de l'ENSTA à Palaiseau, sera une installation conçue pour mener ce genre d'investigation nécessitant un grand nombre de tirs. Basée sur un laser de pompe Ti:Sa à dérive de fréquence capable de délivrer $40 \mathrm{~J}$ avant compression, LASERIX pourra produire des lasers X-UV transitoires de longueurs d'onde comprises entre 7 et $40 \mathrm{~nm}$ à la cadence de $0.1 \mathrm{~Hz}$ (6 tirs par minute).

Outre une amélioration considérable de la cadence des tirs, la technologie "saphir dopé titane" présente de nombreux autres avantages pour une installation destinée à la production de sources $\mathrm{X}$ et X-UV. En comprimant des petites parties du faisceau initial dans des impulsions de $50 \mathrm{fs}$, on pourra multiplier les lignes de lumière X-UV cohérente et réaliser une grande variété d'expériences de type pompe-sonde utilisant des sources X-UV et infrarouge.

La figure 5 présente de façon schématique la future salle d'expérience de LASERIX. La partie "laser" est confinée à l'extrémité de la pièce et peu visible sur la figure. L'enceinte d'interaction dans

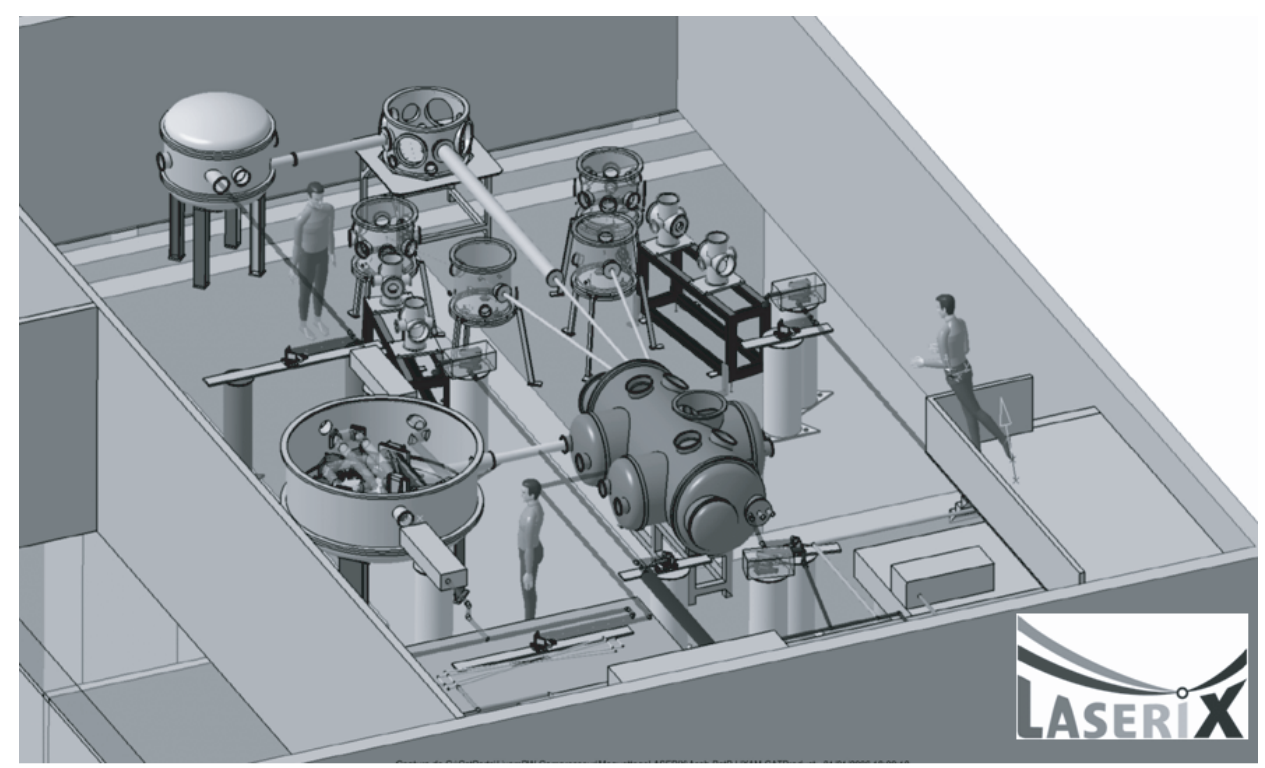

Figure 5. Représentation de la salle d'expériences de LASERIX, station de travail dédiée aux développement des lasers à rayons X-UV et de leurs applications, qui sera mise en service en 2007. 
laquelle sera produit le plasma émetteur de lasers à rayons X-UV occupe le centre de la pièce. Elle contient le dispositif de cible et le système de focalisation de type GRIP [12]. Le faisceau laser X-UV sera ensuite dirigé vers diverses enceintes d'utilisation.

Grâce à cette nouvelle installation, il sera possible de reproduire l'expérience du PALS non plus pour démontrer la faisabilité de la méthode, mais pour analyser de façon exhaustive le comportement des surfaces optiques sous champ laser et mieux comprendre les phénomènes observés au cours de ces premières observations.

\section{Remerciements}

Ce travail a bénéficié du soutien de la fondation scientifique tchèque $\mathrm{N}^{\circ}$ 202/05/2316. Par ailleurs, il a été rendu possible grâce au support financier accompagnant l'accès européen accordé à ce programme scientifique dans le cadre du 6ème PCRD (Contrat RII3-CT-2003-506350). De plus, un des auteurs (K. Cassou) a bénéficié d'une bourse de séjour de recherche « Marie-Curie » (Contrat HPMT-CT-2001-00263). Nous remercions vivement Henri Bercegol (CEA CESTA) pour des discussions très fructueuses, Jean-Jacques Fermé (SESO, Aix-en-Provence) qui nous a fourni gracieusement une partie des échantillons.

\section{Références}

[1] F. Albert, D. Joyeux, P. Jaeglé, A. Carillon, J. P. Chauvineau, G. Jamelot, A. Klisnick, J.C. Lagron, D. Phalippou, D. Ros, S. Sebban and Ph. Zeitoun, Opt. Comm. 142, 184, (1997).

[2] Ph. Zeitoun, F. Albert, P. Jaeglé, D. Joyeux, M. Boussoukaya, A. Carillon, S. Hubert, G. Jamelot, A. Klisnick, D. Phalippou, J. C. Lagron, D. Ros, S. Sebban and A. ZeitounFakiris, Nucl. Instr. and Meth. in Physics Research. A., 416 (1), 189, (1998).

[3] F. Albert, P. Zeitoun, P. Jaeglé, D. Joyeux, M. Boussoukaya, A. Carillon, S. Hubert, G. Jamelot, A. Klisnick, D. Phalippou, D. Ros and A. Zeitoun-Fakiris, Phys. Rev. B -Condensed-Matter, 60 (15), 11089, (1999).

[4] B. Rus, T. Mocek, A. R. Präg, M. Koslová, G. Jamelot, A. Carillon, D. Ros, D. Joyeux, D. Phalippou, Phys. Rev. A 66, 063805 (2002).

[5] B. Rus, T. Mocek, A. R. Präg, M. Kozlová, M. Hudecek, G. Jamelot, A. Carillon, D. Ros, J. C. Lagron, D. Joyeux and D. Phalippou, Plasma Phys. Control. Fusion 44, B207-23, (2002).

[6] G. Jamelot, D. Ros, A. Carillon, B. Rus, T. Mocek, M. Kozlová, A. R Präg, D. Joyeux, D. Phalippou, M. Boussoukaya, M. Kalmykow, F. Ballester and E. Jacques, J. of Applied Physics 98, 044308, (2005).

[7] H. Tang, O. Guilbaud, G. Jamelot, D. Ros, A. Klisnick, D. Joyeux, D. Phalippou, M. Kado, N. Nishikino, K. Sukegawa, M. Ishino, K. Nagashima, H. Daido, Appl. Phys. B 78, 975, (2004) 1, App. Phys. B, 954 (2004).

[8] T. Mocek, B. Rus, A. R. Präg, M. Kozlová, G. Jamelot, A. Carillon, and D. Ros, J. Opt. Soc. Am. B 20, 1386-1391, 2003.

[9] B. Rus, G. Jamelot, H. Bercegol, M. Kozlova, T. Mocek, P. Homer, J. Polan, M. Stupka, K. Cassou, S. Kazamias, A. Klisnick, D. Ros, C. Danson and S. Hawkes, SPIE 2005.

[10] G. Jamelot, D. Ros, A. Klisnick, Ph. Zeitoun, J. Dubau, J.C. Lagron, L. Vanbostal, J. Phys. IV France 108 (2003) 165-8.

[11] G. Jamelot, D. Ros, M. Pittman, A. Klisnick, J-C. Lagron, Ph. Zeitoun, J. Dubau, F. Plé, D. Benredjem, S. Kazamias, O. Guilbaud, J-P. Chambaret, X-Ray Lasers 2004, IOP Conf Series (2006).

[12] S. Kazamias et al., dans ces comptes-rendus. 